Journal of Case Reports 2018;8(3):219-222

\title{
Intraoperative Breakage of LMA Supreme in Anticipated Difficult Airway
}

\author{
Anupriya Saxena, Madhu Gupta, Shikha Chahar, Chitrambika P \\ Department of Anesthesiology and Critical Care, ESI-PGIMSR, Basaidarapur, New Delhi, India.
}

Corresponding Author:

Dr. Anupriya Saxena

Email: anupriya.pmch@gmail.com

This is an Open Access article distributed under the terms of the Creative Commons Attribution License (creativecommons.org/ licenses/by/3.0).

Received

Accepted

Published

July 20, 2017

September 12, 2018

September 20, 2018

\begin{abstract}
Background: The laryngeal mask airway (LMA) Supreme is a single-use, secondgeneration supra-glottic airway device that gained popularity because of its ease of insertion and higher sealing pressure. We report a case of intra-operative LMA supreme breakage in a patient with anticipated difficult airway. Case Report: A 41 year-old female patient with anticipated difficult airway was posted for modified radical mastectomy under general anesthesia utilizing LMA Supreme. After one hour of uneventful surgery significant air leak was noticed. After excluding all possible causes of air leak, while repositioning the LMA, we noticed a break in LMA. The airway was successfully rescued with an endotracheal tube (ETT) rail-roaded over a bougie inserted through the broken end of the LMA. The breakage could be attributed to inadvertently exerted pressure and remained unnoticed because the LMA was covered by surgical drapes. Conclusion: Continuous vigilance and preparedness to handle difficult airways throughout the procedure are essential to manage such cases.
\end{abstract}

Keywords: General Anaesthesia, Glottis, Intratracheal Intubation, Modified Radical Mastectomy.

\section{Introduction}

The LMA Supreme is an innovative, single use supraglottic airway device. It has a rigid moulded proximal component which forms separate airway and drain tube ports, this component is fixed to the bite block. The LMA Supreme is indicated for securing the airway in routine and emergency anaesthetic procedures and also as a rescue airway device in CPR procedures and in difficult airway.

\section{Case Report}

A41-year-old woman (ASA grade, 1) was scheduled to undergo modified radical mastectomy. Airway examination revealed a heavy jaw, mouth opening of $2.0 \mathrm{~cm}$, and Mallampati class III. The patient's thyromental distance and neck movements were normal. The surgery was planned under general anesthesia using LMA Supreme.
Standard monitoring was initiated and baseline parameters were recorded. The LMA Supreme was thoroughly inspected for visible cracks or leaks, and a strict pre-use check was performed according to the manufacturer's instructions. Anesthesia was induced with $1 \mathrm{mcg} /$ $\mathrm{kg}$ of fentanyl and $2 \mathrm{mg} / \mathrm{kg}$ of propofol and a ventilation check was performed Single-hand ventilation was not .possible due to heavy jaw, so a two-hand technique was used with Guedel airway. When the patient showed adequate chest expansion, $0.1 \mathrm{mg} / \mathrm{kg}$ of vecuronium was administered followed by mask ventilation. LMA Supreme size 3 was successfully inserted in a single attempt after achieving an adequate anesthetic plane; the cuff was then inflated with $20 \mathrm{ml}$ of air and an intracuff pressure of $60 \mathrm{~cm} \mathrm{H}_{2} \mathrm{O}$. The cuff position was confirmed by squarewave form capnography. All post-insertion tests were performed, and a 14-Fr 
Ryle's tube was inserted through the gastric port. The LMA Supreme was then meticulously fixed in an erect position. Anesthesia was maintained using $2 \%$ end-tidal sevoflurane with nitrous oxide and oxygen to an MAC of 1 . Pectoral nerves block 1 and 2 blocks were administered under ultrasound guidance for intra-operative and postoperative pain relief. The patient was ventilated in the volume control mode using the Blease Sirius Spacelabs anesthesia workstation. The end-tidal carbon dioxide concentration was maintained at $35-45 \mathrm{mmHg}$.

After about an hour of surgery, the bellows suddenly collapsed, the $\mathrm{EtCO}_{2}$ curve started dampening, peak pressure decreased, and a significant leak was suspected. We immediately performed checks for cuff leakage, disconnected breathing circuits, loosened $\mathrm{CO}_{2}$ canisters, and malfunctioning unidirectional valves, but we did not notice any of these issues. We switched to manual bag ventilation, which increased the flow but still did not allow proper ventilation, even with a fully closed adjustable pressure limiting valve. LMA repositioning was attempted subsequently, and during repositioning, the LMA was found to be broken with significant air leakage from it. Considering the possibility of a difficult airway, we decided to secure the patients airway using a bougie first. Insertion of a hollow gum-elastic bougie was attempted from the broken end of the LMA. Although we initially encountered resistance at the junction of the airway tube and the pre-curved cuff, we were able to place the bougie successfully [Fig.1]. The broken LMA was then removed over the bougie. Throughout the procedure, the patient was ventilated with $100 \%$ oxygen through the hollow bougie. The airway was secured by railroading an endotracheal tube (ETT; internal diameter, $7.5 \mathrm{~mm}$ ) over the bougie, and its position was confirmed by the square capnographic waveform. Meanwhile, the patients' vital parameters were checked carefully, and the patient showed no adverse sequelae with an oxygen

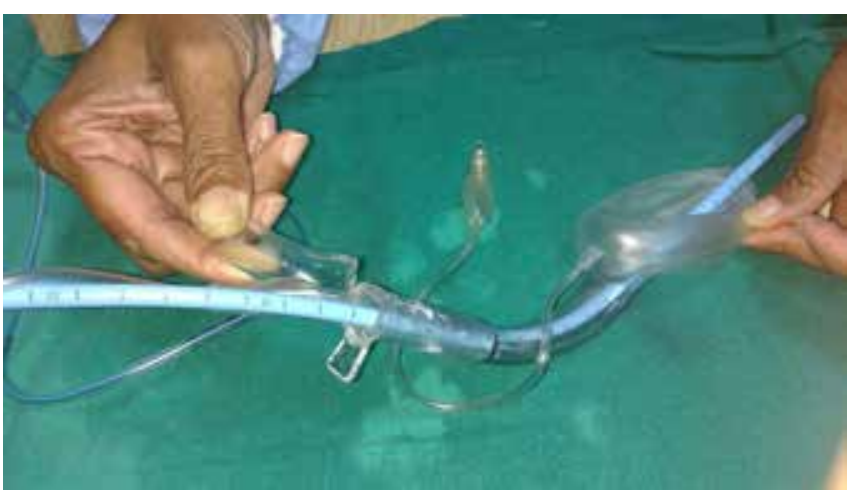

Fig.1: Bougie insertion through broken end of LMA.

saturation of $100 \%$ throughout the procedure. Anesthesia was successfully reversed; the patient was conscious and properly oriented and was then shifted to recovery.

\section{Discussion}

The LMA Supreme is a single-use, secondgeneration airway device optimal for controlled ventilation at high ventilatory pressures [1] and can simplify the optimal insertion technique. LMAs have immeasurably improved our ability to handle difficult airways and can serve as both primary airways and as guide to intubation in difficult airways [2-4].

Intra-operative leaks can occur because of various reasons, such as disconnection of the anesthesia breathing circuit, deflation of the LMA cuff or LMA displacement, or leaks in the ventilator, $\mathrm{CO}_{2}$ canister, unidirectional valves, breathing circuit, $\mathrm{Y}$ piece, and APL valve. A number of machine-related factors and humanmachine interactions influence such situations. The daily pre-anesthesia check out of the anesthesia machine, based on the 2008 recommendations, and the manufacturer-recommended automatic self-check had been done, the airway tubing was adequately checked, and the LMA was secured well with no bending and dragging or traction of the breathing circuit. Therefore, these factors were ruled out in our case. A quick check for all possible connections was also done to rule out any 
accidental disconnection as the cause of the air leak.

The LMA Supreme obtained from the patient showed a break at the junction of the bite block with the airway and drain tube. The LMA Supreme did not show any visual cracks or cuff leaks prior to insertion, and the device was introduced strictly according to the manufacturer's guidelines. After locating the site of the leak, the other options for effective ventilation were as follows: i) removal of the damaged LMA and bag-mask ventilation, but bag-mask ventilation was difficult in our case, so we did not choose this option; ii) replacement of the damaged LMA with a fresh LMA. However, we did not have another LMA of the appropriate size at that time; iii) introduction of an ETT connector connecting the damaged and normal portions of the airway tube. We attempted this approach by passing a $7.5 \mathrm{~mm}$ ETT connector, but it was compressing the drain tube. Since the Ryle's tube was inserted through the drain tube and was getting compressed, we discontinued this procedure; iv) insertion of an ETT into the LMA shaft and inflation of the ETT cuff to form a seal between it and the LMA. We inserted a $4.0 \mathrm{~mm}$ portex cuffed ETT, but we were quite apprehensive about ventilating the patient with this new method since the ETT was very small in size and was difficult to secure in place.

Simon BP have also reported intra-operative breakage of the LMA Supreme on its first use. The exact cause could not determined in their case [5]. After thorough evaluation in this case, we concluded that since the surgical site was close to the airway and the LMA was fixed in an erect position and was completely covered under surgical drapes, LMA breakage could have been caused by in advertent pressure exerted by the surgeons standing very close to the head end during axillary dissection, and the assistant surgeon's hand jerked suddenly due to malpositioning of the surgical retractor.

Although there have been a few cases of ETT disconnection under surgical drapes, to our knowledge, no study to date has reported LMA breakage under surgical drapes. Rose G et al. reported a unusual case of breathing system disruption under circumferential plastic drapes in facial surgery [6]. Yadav $\mathrm{M}$ et al. and Wong DT et al. reported the hazards of excessive LMA reuse leading to fracture of the LMA shaft [7,8], but the LMA Supreme is a single-use device and was hence not reused. Spielman FJ has reported complete separation of tube from mask during removal of a disposable laryngeal mask airway. Yamaguchi $S$ et al. reported damage of a laryngeal mask airway before the start of operation hence emphasizing on importance of peruse inspection. In our case also we strictly followed the manufacturers guidelines in terms of peruse check, size of LMA used, cuff inflation volume, insertion, fixation technique and the expiry date of LMA $[9,10]$.

\section{Conclusion}

Vigilance is the price of safety. Anaesthesiologists should be vigilant in surgeries close to the airway. Whenever possible the LMA must be visible to the anaesthesiologist throughout the surgery with help of surgical screen and patients head should preferably be turned away from the operative site. Many a times supra-glottic device are used in difficult airway situations, where immediate airway control with an alternative method can be difficult and any such complication can result in an nightmare for the anaesthesiologist.

Contributors: AS: designed and drafted the work; MD: revising it critically for important intellectual content; SC, $\mathrm{CP}$ : editing and critical inputs into the manuscript. AS will act as a guarantor. All authors approved the final version of this manuscript.

Funding: None; Competing interests: None stated.

\section{References}

1. Supreme TM. LMA Instruction Manual. Venner Medical, Singapore: Pte Ltd; April 2012.

2. Ramachandran K, Santhanagopalan K. Laryngeal mask airway and the difficult airway. Current Opinion in Anaesthesiology 2004;17(6):491-493. 
3. Ghaus M. Laryngeal mask airway supreme TM for difficult airway management and establishing ventilation in the intensive care unit. Indian J Anaesth. 2014;58: 91-93.

4. Siddiqui S, Seet E, Chan WY. The use of laryngeal mask airway Supreme ${ }^{\mathrm{TM}}$ in rescue airway situation in the critical care unit. Singapore Med J. 2014;55:205-06.

5. Simon BP. A case of breakage of LMA Supreme on its first use. Indian J Anaesth. 2011;50:635-636.

6. Rose G, Eichhorn J, Lexington. Wrap delays detection of disconnect. The Official Journal of the Anesthesia Patient Safety Foundation. 2007;22(2):36.
7. Yadav M, Sandeep G, Mahesh R, Gopinath R. Fractured laryngeal mask airway: Hazards of excessive reuse. Anesth Essays Res. 2015;9:453-454.

8. Wong DT, McGuire GP. Fractured laryngeal mask airway. Can J Anaesth. 2000;47:716.

9. Spielman FJ. Complete separation of the tube from the mask during removal of a disposable laryngeal mask airway. Can J Anesth. 2002;49:990-992.

10. Yamaguchi S, Mishio M, Okuda Y, Kitajima T. Damage of a laryngeal mask airway during anesthesia. Masui. 2000;49:762-764. 